\title{
Efficiency and risks of one-anastomosis gastric bypass
}

\author{
Rene Aleman, Emanuele Lo Menzo, Samuel Szomstein, Raul J. Rosenthal \\ Department of General Surgery, Section of Minimally Invasive Surgery, The Bariatric \& Metabolic Institute, Cleveland Clinic Florida, Weston, \\ FL, USA \\ Contributions: (I) Conception and design: R Aleman; (II) Administrative support: E Lo Menzo, RJ Rosenthal; (III) Provision of study materials or \\ patients: R Aleman, S Szomstein; (IV) Collection and assembly of data: R Aleman; (V) Data analysis and interpretation: R Aleman, E Lo Menzo; (VI) \\ Manuscript writing: All authors; (VII) Final approval of manuscript: All authors. \\ Correspondence to: Raul J. Rosenthal, MD, FACS, FASMBS. Chairman, Department of General Surgery and Director, Bariatric and Metabolic \\ Institute, Cleveland Clinic Florida, 2950 Cleveland Clinic Blvd, Weston, FL 33331, USA. Email: ROSENTR@CCF.ORG.
}

\begin{abstract}
The single-anastomosis gastric bypass has been proposed as a simpler and efficient weight loss reducing surgery. Postoperative outcomes are comparable to those of contemporary popular procedures. There are, however, controversies regarding the efficiency and risks of one-anastomosis gastric bypass (OAGB). The purpose of this review is to define the role of OAGB in metabolic surgery via its operative outcomes. A review of English language literature was performed using the PubMed database, basing the search on the following keywords: "one-anastomosis gastric bypass" AND "outcomes". A total of 238 articles were considered for review. Following thorough screening and selection criteria, 7 articles were considered sufficient for assessment. The nature of the available evidence of this technique poses a challenge to OAGB in its establishment as a standard of care procedure. The anatomical configuration following surgery, as well as the metabolic implications of its hypo-absorptive nature, raises controversial and ongoing concerns that are yet to be addressed. Hence, prospective studies with long-term follow-up ( $>5$ years) can bypass these concerns and allow the progression of the clinical practice of OAGB.
\end{abstract}

Keywords: One-anastomosis gastric bypass (OAGB); single anastomosis gastric bypass; mini gastric bypass; weight loss; obesity; bariatric surgery

Submitted Sep 29, 2019. Accepted for publication Jan 16, 2020.

doi: 10.21037/atm.2020.02.03

View this article at: http://dx.doi.org/10.21037/atm.2020.02.03

\section{Introduction}

With the increase in the prevalence of obesity worldwide, a parallel need to satisfy the therapeutic options for the management of this disease has developed. In 2016, the prevalence of obesity in the US was $39.8 \%$ and affected roughly 93.3 million adults (1). To illustrate the upward trend in the surgical management of obesity, the "Guidelines for the management of overweight and obesity in adults" from the American College of Cardiology, the American Heart Association, and the Obesity Society have stated that $64.5 \%$ of American adults are recommended to undergo weight loss treatment (2). Up to 32 million patients, then, could be considered for bariatric surgery.

Current practice of bariatric surgery in the US places both laparoscopic vertical sleeve gastrectomy (LVSG) and laparoscopic Roux-en-Y gastric bypass (LRYGB) as the most commonly performed procedures. According to the American Society of Metabolic and Bariatric Surgery (ASMBS), the latest yearly estimate of bariatric surgeries in 2017 was 228,000 , of which $59.39 \%$ and $17.80 \%$ corresponded to LVSG and LRYGB, respectively (3). Despite their popularity, the one-anastomosis gastric bypass (OAGB) has emerged as a new alternative for the surgical management of obesity. Since 2011, OAGB has become an increasingly prevalent bariatric surgical procedure across the globe, and represented $1.5 \%$ of all surgeries performed (4). With the growing expectations of this procedure and the undeniable increment in its practice, we present a thorough evaluation of OAGB, its outcomes, effectiveness, and risks. 


\section{History}

The OAGB was previously referred to as the 'mini' gastric bypass or the single-anastomosis gastric bypass. However, the International Federation for the Surgery of Obesity and Metabolic Disorders (IFSO) has agreed that the standard nomenclature for this procedure should be the mini gastric bypass-one anastomosis gastric bypass (MGB-OAGB) (5). The concept behind a single anastomosis or 'loop' gastric bypass was first described by Mason and Ito in 1967 (6). In the original configuration, the authors described a short and wide gastric pouch, horizontally based, and a loop gastrojejunostomy at the lower end of the pouch. Due to the reflux-inducing nature of this procedure, this concept was quickly disregarded as a viable option. It was Rutledge who modified this configuration in 1997 and named it 'mini gastric bypass' (MGB), because the procedure was performed through a 'mini-laparotomy' (7).

The MGB had the particularity of a long-sleeved gastric pouch made from the lesser curvature, which extends proximally toward the angle of His. The gastrojejunostomy consisted of an antecolic 3 to $5 \mathrm{~cm}$ wide anastomosis about 180 to $220 \mathrm{~cm}$ from the ligament of Treitz. The distance of the loop would be $250 \mathrm{~cm}$ in the super obese, 180 to $200 \mathrm{~cm}$ in the elderly or vegetarians, and $150 \mathrm{~cm}$ in patients with type 2 diabetes (8).

Similarly, Carbajo and Caballero proposed a technical variation in 2002. In their version, called one anastomosis gastric bypass, the intention was to prevent the imposed gastroesophageal (GE) bile reflux. This was achieved by a latero-lateral anastomosis between the jejunum and the gastric pouch, with a distance to the ligament of Treitz of about 250 to $350 \mathrm{~cm}$ (9).

Since then, an increasing number of surgeons worldwide are now performing OAGB as a primary surgical procedure for obesity. At the time, the lack of consensus in regard to this procedure was hindering progress. Thus, a consensus statement was performed using a modified Delphi approach on the operative aspects of OAGB. The committee involved 101 recognized experts in bariatric surgery with specialized experience in OAGB. The experts from 39 countries were invited to vote on 55 statements in controversial subjects or variations associated with this procedure. Agreement amongst $\geq 70 \%$ of the experts was indicative of consensus. A consensus was achieved in 48 of the 55 proposed statements and it was concluded that, despite these efforts, there are several areas of disagreement that persist and require further evaluation (10).
The ASMBS has granted the appraisal to OAGB as a technique with relatively short operative time, low complication rates, and excellent weight loss outcomes (11). The current available evidence regarding OAGB extends to more than 70 publications with over 38,000 patients, including review articles, clinical studies, and randomized controlled trials (11-15). In spite of its popularity, mainly in Europe and the Asia Pacific regions, its practice has migrated slowly to the US as a promising bariatric alternative. Hence, it is of utmost importance to recognize its outcomes, advantages, efficiencies, and risks.

\section{Technique}

Considering the multiple descriptions for this technique, the operative technique can be best divided in three main sections: (I) gastric pouch; (II) gastrojejunostomy; (III) limb length.

\section{Gastric pouch}

Firstly, the patient is positioned in a modified lithotomy. Similar to other contemporary bariatric procedures, OAGB is performed laparoscopically with 6 trocars. Usually, there should be one camera port and two $12-\mathrm{mm}$ ports, and the remaining ports should be $5 \mathrm{~mm}$. Upon access to the abdominal wall, the creation of a tubular gastric pouch from the lesser curvature is achieved. This is done by stapling the stomach from the body junction-antral junction toward the angle of His. Although there is a discrepancy regarding the starting point for gastric stapling, there appears to be a tendency to initiate at the level of or directly below the Crow's foot on the lesser curvature. Much like when performing an LVSG, an endoluminal bougie is passed through to calibrate the pouch prior to complete resection. The majority of the studies describe the technique for OAGB using a 36 French bougie; nevertheless, the size has varied in diameter, from a $1-\mathrm{cm}$ nasogastric tube to a 41 French bougie $(5,11,16,17)$.

\section{Gastrojejunostomy}

Following the formation of the gastric pouch, a jejunal loop, roughly $200 \mathrm{~cm}$ from the ligament of Treitz, is brought cephalad in an antecolic/antegastric fashion to create the loop gastrojejunostomy. The rationale of the length of the limb came as a result of the first 209 patients on which OAGB was performed. Initially, the entire small bowel 


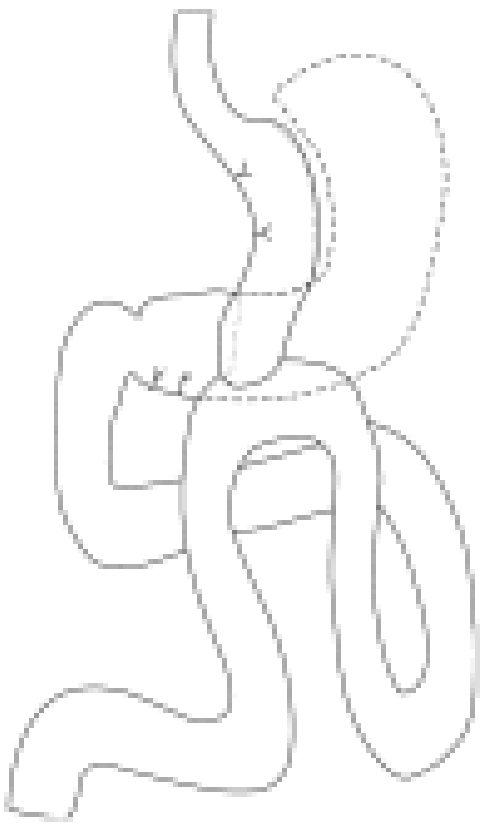

Figure 1 Postoperative upper gastrointestinal tract fluoroscopic study showing the gastrojejunostomy after OAGB. OAGB, oneanastomosis gastric bypass.

was measured from the ligament of Treitz to the ileocecal valve in order to determine a standardized measure for both afferent and common channel. It was observed that the midportion ranged from 250 to $350 \mathrm{~cm}$, rendering a similarity in the lengths of the limbs (9). A linear staple is used for stapling of the anastomosis, with a varying length of 30 to $60 \mathrm{~mm}$ (5). Completely hand-sewn anastomoses have been described; however, the stapled technique seems to be the preferred approach (18-20) (Figure 1). Figure 2 depicts the typical appearance of an UGI test after OAGB.

\section{Limb lengtb}

Two reviews on the performance of OAGB have demonstrated that, on average, a limb length of $200 \mathrm{~cm}$ has been used $(5,11)$. However, in a trial performed by Carbajo et al. on OAGB outcomes in 1,200 patients, the length of the limb was tailored according to the candidates' BMI and comorbidities. For increasing BMIs, an additional 10 to $50 \mathrm{~cm}$-with no specific formula or rationale—of afferent limb was added, maintaining a range of 250 to $300 \mathrm{~cm}$ of common channel (16). Similarly, Lee and colleagues adjusted the afferent limb based on the preoperative BMI. In contrast to Carbajo's outcomes, Lee et al. found

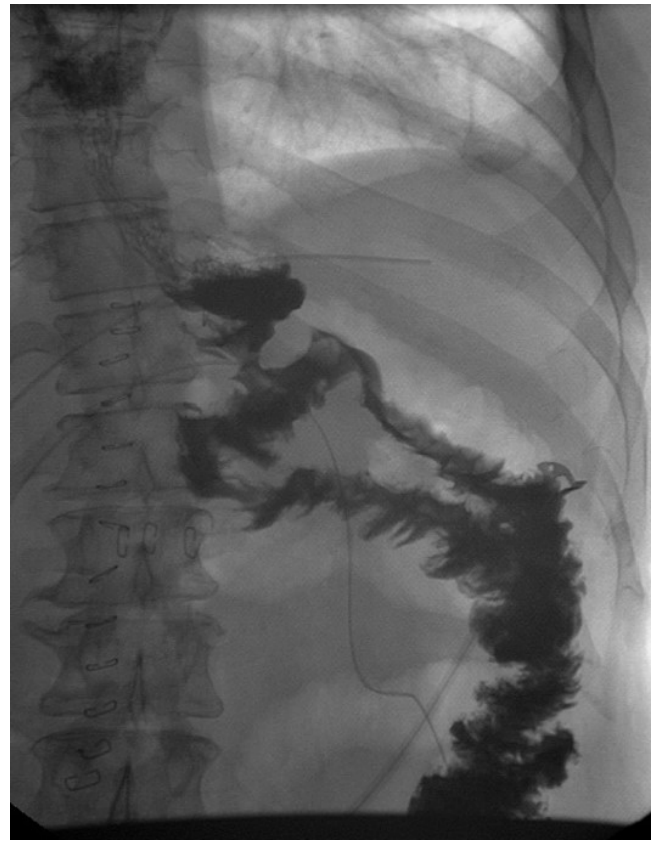

Figure 2 Diagram showing the final anatomy after OAGB. OAGB, one-anastomosis gastric bypass.

excellent weight loss results in all BMI stratifications with limb lengths ranging from $150 \mathrm{~cm}$ for patients with a BMI $<40 \mathrm{~kg} / \mathrm{m}^{2}, 250 \mathrm{~cm}$ for patients with a BMI $40-50 \mathrm{~kg} / \mathrm{m}^{2}$, and $350 \mathrm{~cm}$ for patients with a BMI $>50 \mathrm{~kg} / \mathrm{m}^{2}(21)$. However, based on the heterogeneous data available on surgical success with different limb lengths, there is still no definite consensus on the ideal limb length. Most studies have shown optimal results with a limb length of $200 \mathrm{~cm}$ from the ligament of Treitz, albeit, recent reports advocate for $150 \mathrm{~cm}(8,17,20,22)$. Ultimately, in lieu of consensus, the decision should be based on a case-dependent manner and on the surgeon's experience.

Intestinal length remains controversial in the literature, with a wide array of results that depend on the technique used. In reference to relevant data, the length of the small bowel ranges from 4.7 to $9.7 \mathrm{~m}$ in vivo (23-27). Some studies have somewhat correlated intestinal length with sex, age, weight, height, or race. The discrepancy has partially established a lack of interest on the subject, to such an extent that contemporary published articles and textbooks are limited. In an anatomical study on the length of the human intestine, 200 non-fixed adult cadavers were studied. Post-mortem average length of the whole intestine was $795 \pm 129 \mathrm{~cm}$, and was significantly longer in men and younger subjects (28). The premise of having awareness of 
Table 1 Prospective cohort studies on OAGB and weight loss

\begin{tabular}{lccr}
\hline Study details & Number of patients & EWL (\%) & Follow-up (\%) \\
\hline Rutledge and Walsh (31) & 2,410 & 80.5 & NR \\
Musella et al. (32) & 838 & $70.1 \pm 8.4$ & 94.8 at 12 months \\
Yang et al. (33) & 89 & $70 \pm 20$ & 100 at 12 months \\
Noun et al. (20) & 126 & 68.4 & 45.2 at 23 months \\
Lee et al. (34) & 1,163 & 72.9 & 56 at 5 years \\
\hline
\end{tabular}

EWL, excess weight loss; NR, not reported; OAGB, one-anastomosis gastric bypass.

the intestinal length is directly related to clinical disorders induced by large intestinal resections. Sizing and level of resection may result in diarrhea and severe nutritional complications due to lack of fat absorption, protein, vitamins, and hydroelectric imbalances caused by lack of water absorption (29). It is well known that a resection of the small bowel with less or equal to $75 \mathrm{~cm}$, will result in "short bowel syndrome" (28). Thus, the standardization of the limb length is of utmost importance to avoid malabsorptive complications secondary to aleatory bowel resection.

\section{Efficiency and risks}

Current data on OAGB identifies this procedure as a safe and feasible, with short operative times, low complication rates, and excellent weight loss outcomes (11). The limited retrospective data and lack of long-term follow-up longer than 5 years hinders present publications. Evidently, the anatomical configuration and malabsorptive characteristics of the OAGB will inevitably generate nutritional deficiencies and bile reflux. These and many more queries are still unanswered, leaving discrepancies on clinical application. Nonetheless, the relative ease in the performance of this surgery has triggered interest among bariatric surgeons. Hence, satisfactory outcomes are possible with a thorough understanding of the implied efficiencies and risks.

\section{Weight loss}

Four randomized controlled trials have reported outstanding weight loss results following primary OAGB. Weight loss was reported at 12 months (EWL $66.9 \% \pm 23.7 \%$ and $66.9 \% \pm 10.9 \%$ ), 2 years (EWL $64.4 \% \pm 8.8 \%$ ), and 5 years (EWL $22.8 \% \pm 5.9 \%)(12,14,15,30)$. Table 1 summarizes a list of prospective cohort studies and their weight loss outcomes. Interestingly, Lee et al. compared OAGB to LVSG in a 5 -year prospective trial. The mean starting BMI was lower than those of other trials at $30.2 \pm 2.2$ and the excess body weight loss (EWL) achieved was over $100 \%$ with a mean finishing BMI of $23.3 \pm 2.2 \mathrm{~kg} / \mathrm{m}^{2}$. These results, however, did not differ significantly in comparative operations (14). The EWL observed in these studies further supports the utilization of OAGB as a suitable bariatric surgical alternative. The comparison of OAGB to other bariatric surgical interventions is discussed later in this chapter.

\section{Morbidity and mortality}

A large prospective trial reporting the outcomes of 1,200 patients who underwent OAGB described the complications and side effects following surgery (16). Complications were divided into those occurring intraoperatively requiring conversion to open surgery, immediate postoperative complications resolved by open reoperations, immediate postoperative complications resolved by re-laparoscopy, early postoperative complications resolved conservatively, major late complications, and other complications and side effects. Intraoperative complications leading to conversions occurred in $0.3 \%$. Overall, complications requiring operations occurred in $1.3 \%$ and resolved conservatively in $1 \%$. This study reported only $2(0.16 \%)$ deaths, which occurred early in the trial.

Comparatively, Carbajo et al.'s first reported outcomes following OAGB resulted in $0.9 \%$ of conversions to open surgery, $1.3 \%$ of immediate postoperative re-operations, and $2.3 \%$ of complications managed conservatively, and had a total of $2(0.8 \%)$ deaths (9). Another study conducted by IFSO reported one early death in-trial in four reviewed randomized controlled trials (3.3\% for that trial specifically, and $0.05 \%$ in general) $(5,14)$. This review further highlights 
Table 2 Possible complications after OAGB

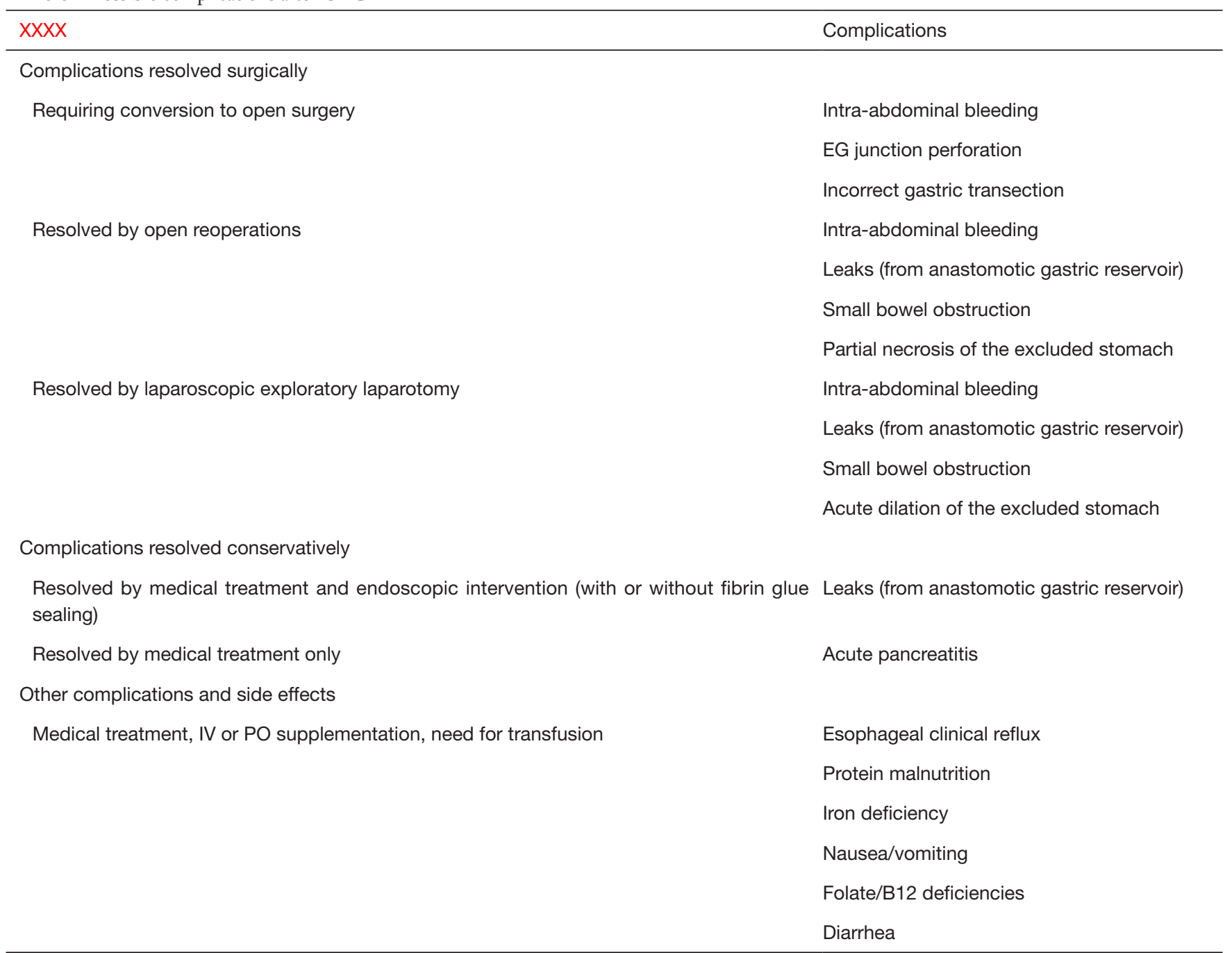

OAGB, one-anastomosis gastric bypass.

15 deaths occurring in prospective and retrospective cohort studies, representing an overall death rate of $0.17 \%$ (20,31-34). Consequently, the gross mortality rates for OAGB range from $0.16 \%$ to $3.3 \%$, while universal complication rates are reported in $0.3 \%$ to $2.3 \%$. The stratification of complication etiologies is summarized in Table 2.

Other than the relative technical implications of OAGB, its malabsorptive aspect determines positive effects on obesity-related comorbidities. Similar to LVSG and LRYGB, there is a variant outcome in the management of comorbidities, in that it may evoke remission or improvement following surgery. The comorbidities that have shown complete resolution or substantial improvement include type 2 diabetes mellitus (T2DM), insulin resistance, hypertension, hyperlipidemia, liver steatosis, and obstructive sleep apnea (16). Improvement was also observed in a significant majority of patients with mechanical complications related to chronic obesity like osteoarthritis and respiratory insufficiency (16).

Remarkably, the management of T2DM and metabolic syndrome has been widely described as a comorbidity of interest in randomized controlled trials. Lee et al. reported a $100 \%$ resolution in the patients $(n=80)$ with metabolic syndrome at 2 years (12). Parallel to these findings, at 5 years, $60 \%$ of participants $(\mathrm{n}=60)$ with T2DM at baseline had an $\mathrm{HbA1c}<6.5 \%$ requiring no medical therapy in a trial 
Table 3 Prospective cohort studies and T2DM changes

\begin{tabular}{lcc}
\hline Study details & Number of patients & T2DM resolution (\%) \\
\hline Bruzzi et al. (35) & 175 & 82 \\
Yang et al. (33) & 89 & HbA1c from $6.5 \pm 1.4$ to \\
Musella et al. (32) & 974 & $5.3 \pm 0.5^{\star}$ \\
Lee et al. (34) & 1,163 & 84.4 \\
Noun et al. (20) & 126 & 89 \\
$\begin{array}{l}\text { Rutledge and } \\
\text { Walsh (31) }\end{array}$ & 2,410 & $85^{\star *}$ \\
\hline
\end{tabular}

*, $\mathrm{P}=0.001 ;{ }^{* *}, \mathrm{~T} 2 \mathrm{DM}$ unspecified. T2DM, type 2 diabetes mellitus; HbA1c, glycosylated hemoglobin.

focused on eliciting change in diabetes after surgery (14). Notwithstanding the inadequacy of metabolic impact following surgery in randomized controlled studies, prospective cohort studies that addressed change in T2DM have reported major improvement after surgery. The results are summarized in Table 3.

A major concern when performing OAGB is the biliary reflux secondary to the creation of the loop gastrojejunostomy. Biliary reflux has been reported in $0.9 \%$ to $4 \%$ of patients and the main concern centers on the potential for malignancy in the stomach and esophagus (16,32,35-38). Until now, OAGB is still considered a highrisk operation for the development of gastroesophageal cancer secondary to this pathology. With this notion, a review of the literature was conducted by Ahmed and Addosari in order to determine the theoretical risk of cancer after OAGB. It was determined that the duration of the gastroesophageal (GE) mucosa exposure to the bile is a main determinant in the pathogenesis and progression of metaplasia that may take up to 20 years to completely develop after surgery. While the available evidence supports a prudent attitude and states that it is reasonable to indicate OAGB for patients over 50 years of age, to confirm or disprove said progression, further follow-up is needed (39).

In defiance of these findings, a small case series of 15 patients that evaluated endoscopic examinations after OAGB did not reveal esophagitis, biliary gastritis, or even the presence of refluxed bile after 1 year of follow-up (40). Similarly, Carbajo et al.'s 1,200-case study revealed that 53\% of the patients had gastroesophageal reflux disease (GERD) and all were relieved after operation (16).

It is important to acknowledge the shortcomings associated with the available data on outcomes after OAGB. Most studies focus on weight loss and T2DM; however, future directives should focus on comparative data toward the more popular bariatric procedures and long-term follow-up.

In comparison to the most commonly practiced bariatric surgeries (SG and RYGB), OAGB seems to be a promising candidate for a rapid weight-loss alternative. A systematic review and meta-analysis recently evaluated the short ( $\leq 30$ days) and long-term mortality ( $\geq 2$ years) in patients following bariatric surgery (RYGB, LABG, BPD, SG). Short-term mortality reported a rate of $0.18 \%$ in over 38 randomized controlled trials (95\% CI: $0.04-0.38 \%$ ). For long-term mortality, on the other hand, a reduction of $41 \%$ in all-cause mortality was observed after surgery (41). In contrast, OAGB's mortality rate has been reported in a range from $0.16 \%$ to $3.3 \%$, and complication rates of $0.3 \%$ to $2.3 \%$.

Glycemic control following bariatric surgery has been described extensively. Multiple randomized controlled trials determined the outcomes of bariatric surgery versus the medical management of T2DM. Seven of these trials had a 2-year follow-up and a remission (full or partial) of T2DM. Remission was observed in $52.5 \%$ of the patients undergoing medical management (risk ratio $(R R)=10$, 95\% CI: 5.5-17.9, $\mathrm{P}<0.001)$. Specifically, LRYGB showed significant effect at 2 years follow-up when compared to medical management, and had a greater decrease of HbA1c (0.9 percentage points, 95\% CI: $0.6-1.1, \mathrm{P}<0.001)$ and fasting blood glucose $(35.3 \mathrm{mg} / \mathrm{dL}, 95 \%$ CI: $13.3-57.3$, $\mathrm{P}=0.002$ ), increase of high-density lipoprotein (HDL) $(12.2 \mathrm{mg} / \mathrm{dL}, 95 \%$ CI: 7.6-16.8, $\mathrm{P}<0.001)$, and decrease of triglycerides (32.4 mg/dL, 95\% CI: 4.5-60.3, $\mathrm{P}=0.02)(42)$. In congruence to these findings, a meta-analysis consisting of 136 studies and 22,094 patients who underwent bariatric surgery (RYGB, LAGB, SG, and BPD) reported an overall $77 \%$ remission of T2DM after bariatric surgery. When stratified by procedure and outcomes, remission was $48 \%$ for laparoscopically adjustable gastric banding (LAGB), $68 \%$ for vertical banded gastroplasty (VBG), $84 \%$ for LRYGB, and 98\% for biliopancreatic diversion (BPD) (43). If the reported overall remission rate of T2DM ranges along $70 \%$ to $85 \%$ in all bariatric procedures, and OAGBs remission rate has been reported in $\geq 80 \%$, it is inferred that OAGB serves as an adequate alternative for the surgical management of this metabolic disease. This holds sound when considering complication rates and weight loss management. 


\section{Short-term outcomes}

One of the most appealing features of OAGB is the expeditious technique. A large case series consisting of 2,140 patients reported a mean operating time (OT) of 37.5 , comparative to the extent it is a shorter average when compared to the original mini-gastric bypass 'thirty-minute case' $(8,31)$. Other large patient cohort trials have reported a mean OT ranging from 86 to 110 minutes (44). There seems to be a parallel association with an increased OT and equally incrementing obesity (21). Nevertheless, certain publications have reported similar OT in patients with a $\mathrm{BMI}>50 \mathrm{~kg} / \mathrm{m}^{2}(45,46)$.

This alternate bariatric procedure presents itself as a promising surgical therapy for rapid weight loss and management of associated comorbidities. Early complication rates range from $3.5 \%$ to $7.5 \%$, and are considered to be acceptable $(16,44)$. Major complicationsas summarized in Table 2-result in reoperation and/or prolonged length of stay, and it has been reported in a rate of $2 \%$ to $3 \%$ of patients undergoing OAGB $(47,48)$. In regards to the appearance of leaks and hemorrhage, the occurrence potential in the early postoperative period following OAGB is possible. The tendency for these events occurs during the first couple of postoperative weeks in $0.7 \%$ to $2 \%$ of the cases $(16,17,20,49)$.

Regarding length of stay, the average ranges from 1 to 5 days postoperatively, with an evident tendency of longer lengths in those patients with higher BMIs or in patients undergoing OAGB as a revisional surgery $(8,12,21,50,51)$.

\section{Long-term outcomes}

The assessment of long-term outcomes after OAGB is somehow limited due to the limited follow-up of both retrospective and prospective available literature. However, weight loss following OAGB appears to be comparable to those of LRYGB, with a pooled percent excess weight loss (\%EWL) of $68.6-85 \%$ at $\geq 5$-year follow-up $(16,32,34,35,52-55)$. The weight loss maintenance compared to that of LVSG has been evaluated by some retrospective series. It is assumed that the retrospective nature and lack of randomization results in variable outcomes, in that some studies have demonstrated superior weight loss with OAGB, while others have reported similar weight loss between OAGB and LVSG $(34,45,46,56)$.

STOPP A randomized controlled trial compared OAGB $(\mathrm{n}=80)$ and LRYGB to determine a difference in $\% \mathrm{EWL}$ at
2 years postoperatively. There was no significant difference in $\% \mathrm{EWL}(64.4 \%$ vs. $60.0 \%)$. Notably, there was a higher operative morbidity in the LRYGB (7.5\% vs. $20 \%$; $\mathrm{P}<0.05)(14)$. A similar randomized controlled trial that consisted of 60 patients undergoing OAGB resulted in higher weight loss at 12 months, when compared to LVSG patients; however, the 5-year follow-up showed non-persistence of said difference $(13,14)$. Taking into consideration that OAGB functions as a metabolic procedure for the resolution of obesity-related comorbidities, the initial mid-term follow-up evidence suggests the similarity in the ability to induce remission of T2DM as other bariatric surgeries $(16,17,31,32,35,57,58)$. A noteworthy set of retrospective series, for example, has reported T2DM remission rates as high as $93.2 \%$ at 6 -year follow-up, and $53 \%$ at 7 years $(52,57,59)$. All of the aforementioned outcomes require further evaluation by longer follow-ups and comparative components to other techniques.

\section{Risks}

The likelihood of complications from this surgical procedure can be compared to those seen in any other metabolic surgery. For instance, the implementation of criteria, psychological evaluation, and preoperative workup to verify if patients are candidates to metabolic surgery are imperative. This preparation is fulfilled in the same manner as other metabolic surgeries, looking for obesityrelated diseases, establishing antithrombotic prophylaxis and treating comorbidities (9). OAGB has a common point among critics regarding the rising concern of gastritis and its potential relation to cancer, illustrating the need for more research studies $(2,3)$.

\section{Complications}

Due to shorter operative time and simplicity of the surgical technique, OAGB has shown fewer early and late surgical complications ranging from $4 \%$ to $7.5 \%$ (11). These complications can be better described as early and late complications.

\section{Early postoperative complications}

\section{Leaks}

Ever since the first publications on OAGB in 2005, new series have shown improvement in outcomes in terms 
of leak rates. This favorable reduction is thought to be secondary to the preservation of vasculature, as a result of partial sectioning and low vascular traction (tension-free anastomosis). While leaks still represent the most common complication of this intervention $(0.1 \%$ to $1.9 \%)$, once a leak is identified, aggressive management strategies must be implemented to avoid further complications $(11,16)$.

\section{Bleeding}

Bleeding is the second most common complication and presents usually in the early postoperative setting. It commonly originates from the staple lining along the gastric pouch, gastric remnant, or gastrojejunal (GJ) anastomosis, with a rate of less than $3 \%$ (11). The performing surgeon must be capable of discerning between an intraluminal and intra-abdominal source of bleeding. This identification must be made prior to attempting any invasive management. The management tends to be conservative, but almost half of patients require surgical intervention $(11,16)$.

\section{Small bowel obstruction}

Internal hernias and small bowel obstruction (SBO) are an uncommon complication seen in OAGB because there are no mesenteric defects to close. This represents a great advantage compared to other metabolic surgeries (i.e., LRYGB). Even though the percentage is significantly low, should the case present itself, diagnosis should be considered $(16,60)$.

\section{Dumping syndrome}

Rapid transit of hyperosmolar content into the jejunum promotes physiologic changes that ultimately result in aggressive GI symptoms. The treatment is the same that is used in other bypass surgeries, mainly focusing on dietary modification (60).

\section{Late postoperative complications}

\section{Stomal stenosis}

Anastomotic size is highly correlated with this type of complication. Case series with an anastomotic size $<2.5 \mathrm{~cm}$ have shown increased incidence rates. Conversely, those who performed anastomotic sizes of $2 \mathrm{~cm}$ present with further problems. Similar to current treatment of stomal stenosis in other bariatric procedures, these are usually treated with pneumatic dilation, and only a small number of patients will require reoperation (16).

\section{Marginal ulcer (MU)}

$\mathrm{MU}$ is a common denominator in most types of bariatric surgery at the level of the GJ junction. It has been hypothesized that the stapling size or an increased gastric acid production in an oversized pouch may cause this lesion. OAGB is thought to have a protective effect as the presence of bile at the GJ level can act as a buffer to avoid the constant injury of the epithelium. In spite of that, the incidence of $\mathrm{MU}$ is around $0.6-4 \%$ for large series, and remains controversial in outcomes following OAGB (60).

\section{Malabsorptive complications}

Due to the anatomical variance of this procedure, malnutrition has been reported in $1.1 \%$ of patients undergoing OAGB (17). Due to this stronger malabsorptive component, OAGB has lower hemoglobin levels than LRYGB (39). Iron deficiency anemia is a common and has a variable incidence from $5 \%$ to $10 \%$ (11). The presence of hypo-absorption and malnutrition has been increasingly reported in patients with an afferent limb $>250 \mathrm{~cm}$ (16). This malnutrition has been described to be transitory, and the majority of patients responded to nutritional supplements without the necessity of immediate intervention or reversal/conversion procedures (39).

\section{Weight loss failure}

Some patients present with an inability to lose weight after the intervention and an excess body mass index loss (EBMIL) $<25 \%$. This can be secondary to dilation of the gastric pouch being diagnosed by upper GI series and should be managed promptly by revisional surgery (17).

\section{Randomized controlled trial}

Due to the relative unpopularity of the OAGB, there is a limited amount of level I evidence- based large controlled trials. Robert et al. recently presented a multicenter, randomized, non-inferiority, controlled trial comparing outcomes of 234 obese patients who underwent either LRYGB or OAGB. After a 2-year follow-up, the mean percentage excess BMI loss was $-87.9 \%$ in the OAGB 
group and $-85.5 \%$ in the LRYGB group. The finding rather confirms non-inferiority of OAGB when compared to LRYGB (mean difference $-3.3 \%$, $95 \%$ CI: $-9.1 \%$ to $2.6 \%)$. Overall, there was no significant difference between the adverse events reported $(\mathrm{P}=0.042)$. Nevertheless, the nutritional complications in the OAGB had a rate of $21.4 \%$, while the LRYGB group reported none $(\mathrm{P}=0.0034)$. As suggested by the authors, the nutritional complications seem to be related to the length of the biliopancreatic limb in the OAGB group, which was set at $200 \mathrm{~cm}$, further worsening the malabsorptive effect encountered in OAGB patients (61). This study has established a precedent in the safety and feasibility of OAGB as a suitable alternate bariatric procedure. It has also highlighted the paramount need to answer the ultimate question in regard to OAGB: "What is the most adequate limb length to ensure successful outcomes?"

\section{Conclusions}

The main goal of bariatric surgery is to manage weight loss and the associated comorbidities. OAGB has a relentless and intriguing appearance in contemporary practice. Its relatively short operative time, low complication rate, excellent weight loss outcomes, and comparative remission/ improvement of obesity-related comorbidities has granted it the role of a viable alternative for operative candidates. The nature of the available evidence of this technique poses a challenge to OAGB in its establishment as a standard of care procedure. The anatomical configuration following surgery, as well as the metabolic implications of its hypo-absorptive nature, raises controversial and ongoing concerns that are yet to be addressed. Hence, prospective studies with longterm follow-up ( $>5$ years) can address these concerns and enable the progression of the clinical practice of OAGB.

\section{Acknowledgments}

Funding: None.

\section{Footnote}

Provenance and Peer Review: This article was commissioned by the Guest Editor (Muhammed Ashraf Memon) for the focused issue "Bariatric Surgery" published in Annals of Translational Medicine. The article was sent for external peer review organized by the Guest Editor and the editorial office.
Conflicts of Interest: The focused issue "Bariatric Surgery" was commissioned by the editorial office without any funding or sponsorship. The authors have no conflicts of interest to declare.

Ethical Statement: The authors are accountable for all aspects of the work in ensuring that questions related to the accuracy or integrity of any part of the work are appropriately investigated and resolved.

Open Access Statement: This is an Open Access article distributed in accordance with the Creative Commons Attribution-NonCommercial-NoDerivs 4.0 International License (CC BY-NC-ND 4.0), which permits the noncommercial replication and distribution of the article with the strict proviso that no changes or edits are made and the original work is properly cited (including links to both the formal publication through the relevant DOI and the license). See: https://creativecommons.org/licenses/by-nc-nd/4.0/.

\section{References}

1. Adult Obesity Facts. Overweight \& Obesity. Available online: https://www.cdc.gov/obesity/data/adult.html

2. Jensen MD, Ryan DH, Apovian CM, et al. 2013 AHA/ ACC/TOS guideline for the management of overweight and obesity in adults: a report of the American College of Cardiology/American Heart Association Task Force on Practice Guidelines and The Obesity Society. Circulation 2014;129:S102-38.

3. Estimate of Bariatric Surgery Numbers, 2011-2017. American Society for Metabolic and Bariatric Surgery. In: American Society for Metabolic and Bariatric Surgery. Available online: https://asmbs.org/resources/estimate-ofbariatric-surgery-numbers

4. Buchwald H, Oien DM. Metabolic/bariatric surgery worldwide 2011. Obes Surg 2013;23:427-36.

5. De Luca M, Tie T, Ooi G, et al. Mini Gastric Bypass-One Anastomosis Gastric Bypass (MGB-OAGB)-IFSO Position Statement. Obes Surg 2018;28:1188-206.

6. Mason EE, Ito C. Gastric Bypass in Obesity. Surg Clin North Am 1967;47:1345-51.

7. Rutledge R, Kular K, Manchanda N. The Mini-Gastric Bypass original technique. Int J Surg 2019;61:38-41.

8. Rutledge R. The Mini-Gastric Bypass: Experience with the First 1,274 Cases. Obes Surg 2001;11:276-80.

9. Carbajo M, García-Caballero M, Toledano M, et al. OneAnastomosis Gastric Bypass by Laparoscopy: Results of 
the First 209 Patients. Obes Surg 2005;15:398-404.

10. Mahawar KK, Himpens J, Shikora SA, et al. The First Consensus Statement on One Anastomosis/Mini Gastric Bypass (OAGB/MGB) Using a Modified Delphi Approach. Obes Surg 2018;28:303-12.

11. Parikh M, Eisenberg D, Johnson J, et al. American Society for Metabolic and Bariatric Surgery review of the literature on one-anastomosis gastric bypass. Surg Obes Relat Dis 2018;14:1088-92.

12. Lee WJ, Yu PJ, Wang W, et al. Laparoscopic Roux-en-Y versus mini-gastric bypass for the treatment of morbid obesity: a prospective randomized controlled clinical trial. Ann Surg 2005;242:20-8.

13. Lee WJ, Chong K, Ser KH, et al. Gastric bypass vs sleeve gastrectomy for type 2 diabetes mellitus: a randomized controlled trial. Arch Surg 2011;146:143-8.

14. Lee WJ, Chong K, Lin YH, et al. Laparoscopic Sleeve Gastrectomy Versus Single Anastomosis (Mini-) Gastric Bypass for the Treatment of Type 2 Diabetes Mellitus: 5-Year Results of a Randomized Trial and Study of Incretin Effect. Obes Surg 2014;24:1552-62.

15. Seetharamaiah S, Tantia O, Goyal G, et al. LSG vs OAGB-1 Year Follow-up Data-a Randomized Control Trial. Obes Surg 2017;27:948-54.

16. Carbajo MA, Luque-de-León E, Jiménez JM, et al. Laparoscopic One-Anastomosis Gastric Bypass: Technique, Results, and Long-Term Follow-Up in 1200 Patients. Obes Surg 2017;27:1153-67.

17. Chevallier JM, Arman GA, Guenzi M, et al. One thousand single anastomosis (omega loop) gastric bypasses to treat morbid obesity in a 7 -year period: outcomes show few complications and good efficacy. Obes Surg 2015;25:951-8.

18. Blanc P, Lointier P, Breton C, et al. The Handsewn Anastomosis with an Absorbable Bidirectional Monofilament Barbed Suture Stratafix® During Laparoscopic One Anastomosis Loop Gastric Bypass. Retrospective Study in 50 Patients. Obes Surg 2015;25:2457-60.

19. Noun R, Zeidan S. Laparoscopic mini-gastric bypass: an effective option for the treatment of morbid obesity. J Chir (Paris) 2007;144:301-4.

20. Noun R, Riachi E, Zeidan S, et al. Mini-gastric bypass by mini-laparotomy: a cost-effective alternative in the laparoscopic era. Obes Surg 2007;17:1482-6.

21. Lee WJ, Wang W, Lee YC, et al. Laparoscopic minigastric bypass: experience with tailored bypass limb according to body weight. Obes Surg 2008;18:294-9.
22. Mahawar KK. A Biliopancreatic Limb of $>150 \mathrm{~cm}$ with OAGB/MGB Is Ill-Advised. Obes Surg 2017;27:2164-5.

23. Ahrens EH Jr, Blankenhorn DH, Hirsch J. Measurement of the Human Intestinal Length In Vivo and Some Causes of Variation. Gastroenterology 1956;31:274-84.

24. Sadahiro S, Ohmura T, Saito T, et al. Relationship between length and surface area of each segment of the large intestine and the incidence of colorectal cancer. Cancer 1991;68:84-7.

25. Sadahiro S, Ohmura T, Yamada Y, et al. Analysis of length and surface area of each segment of the large intestine according to age, sex and physique. Surg Radiol Anat 1992;14:251-7.

26. Saunders BP, Phillips RKS, Williams CB. Intraoperative measurement of colonic anatomy and attachments with relevance to colonoscopy. Br J Surg 1995;82:1491-3.

27. Treves F. Lectures on the Anatomy of the Intestinal Canal and Peritoneum in Man. BMJ 1885;1:415-9.

28. Hounnou G, Destrieux C, Desmé J, et al. Anatomical study of the length of the human intestine. Surg Radiol Anat 2002;24:290-4.

29. Warwick R, Williams PL, Dyson M, et al. "Gray's Anatomy." Br J Radiol 1985;58:1029.

30. Darabi S, Talebpour M, Zeinoddini A, et al. Laparoscopic gastric plication versus mini-gastric bypass surgery in the treatment of morbid obesity: a randomized clinical trial. Surg Obes Relat Dis 2013;9:914-9.

31. Rutledge R, Walsh TR. Continued Excellent Results with the Mini-Gastric Bypass: Six-Year Study in 2,410 Patients. Obes Surg 2005;15:1304-8.

32. Musella M, Susa A, Greco F, et al. The laparoscopic minigastric bypass: the Italian experience: outcomes from 974 consecutive cases in a multicenter review. Surg Endosc 2014;28:156-63.

33. Yang PJ, Lee WJ, Tseng PH, et al. Bariatric surgery decreased the serum level of an endotoxin-associated marker: lipopolysaccharide-binding protein. Surg Obes Relat Dis 2014;10:1182-7.

34. Lee WJ, Ser KH, Lee YC, et al. Laparoscopic Rouxen-Y Vs. Mini-gastric Bypass for the Treatment of Morbid Obesity: a 10-Year Experience. Obes Surg 2012;22:1827-34.

35. Bruzzi M, Rau C, Voron T, et al. Single anastomosis or mini-gastric bypass: long-term results and quality of life after a 5-year follow-up. Surg Obes Relat Dis 2015;11:321-6.

36. Musella M, Susa A, Manno E, et al. Complications Following the Mini/One Anastomosis Gastric Bypass 
(MGB/OAGB): a Multi-institutional Survey on 2678 Patients with a Mid-term (5 Years) Follow-up. Obes Surg 2017;27:2956-67.

37. Saarinen T, Räsänen J, Salo J, et al. Bile Reflux Scintigraphy After Mini-Gastric Bypass. Obes Surg 2017;27:2083-9.

38. Theisen J, Peters JH, Fein M, et al. The mutagenic potential of duodenoesophageal reflux. Ann Surg 2005;241:63-8.

39. Guirat A, Addossari HM. One Anastomosis Gastric Bypass and Risk of Cancer. Obes Surg 2018;28:1441-4.

40. Tolone S, Cristiano S, Savarino E, et al. Effects of omegaloop bypass on esophagogastric junction function. Surg Obes Relat Dis 2016;12:62-9.

41. Cardoso L, Rodrigues D, Gomes L, et al. Short- and longterm mortality after bariatric surgery: A systematic review and meta-analysis. Diabetes Obes Metab 2017;19:1223-32.

42. Khorgami Z, Shoar S, Saber AA, et al. Outcomes of Bariatric Surgery Versus Medical Management for Type 2 Diabetes Mellitus: a Meta-Analysis of Randomized Controlled Trials. Obes Surg 2019;29:964-74.

43. Buchwald H, Avidor Y, Braunwald E, et al. Bariatric surgery: a systematic review and meta-analysis. JAMA 2004;292:1724-37.

44. Mahawar KK, Jennings N, Brown J, et al. "Mini" gastric bypass: systematic review of a controversial procedure. Obes Surg 2013;23:1890-8.

45. Plamper A, Lingohr P, Nadal J, et al. Comparison of mini-gastric bypass with sleeve gastrectomy in a mainly super-obese patient group: first results. Surg Endosc 2017;31:1156-62.

46. Madhok B, Mahawar KK, Boyle M, et al. Management of Super-super Obese Patients: Comparison Between Mini (One Anastomosis) Gastric Bypass and Sleeve Gastrectomy. Obes Surg 2016;26:1646-9.

47. Wang W, Wei PL, Lee YC, et al. Short-term results of laparoscopic mini-gastric bypass. Obes Surg 2005;15:648-54.

48. Piazza L, Ferrara F, Leanza S, et al. Laparoscopic minigastric bypass: short-term single-institute experience. Updates Surg 2011;63:239-42.

49. Georgiadou D, Sergentanis TN, Nixon A, et al. Efficacy and safety of laparoscopic mini gastric bypass. A systematic review. Surg Obes Relat Dis 2014;10:984-91.

50. Noun R, Zeidan S, Riachi E, et al. Mini-gastric bypass for revision of failed primary restrictive procedures: a valuable option. Obes Surg 2007;17:684-8.

51. Wang W, Huang MT, Wei PL, et al. Laparoscopic minigastric bypass for failed vertical banded gastroplasty. Obes Surg 2004;14:777-82.

52. Kular KS, Manchanda N, Rutledge R. A 6-year experience with 1,054 mini-gastric bypasses-first study from Indian subcontinent. Obes Surg 2014;24:1430-5.

53. Noun R, Skaff J, Riachi E, et al. One thousand consecutive mini-gastric bypass: short- and long-term outcome. Obes Surg 2012;22:697-703.

54. Peraglie C. Laparoscopic mini-gastric bypass in patients age 60 and older. Surg Endosc 2016;30:38-43.

55. Sheikh L, Pearless LA, Booth MWC. Laparoscopic Silastic Ring Mini-Gastric Bypass (SR-MGBP): Up to 11-Year Results from a Single Centre. Obes Surg 2017;27:2229-34.

56. Magouliotis DE, Tasiopoulou VS, Tzovaras G. One Anastomosis Gastric Bypass Versus Roux-en-Y Gastric Bypass for Morbid Obesity: An Updated Meta-Analysis. Obes Surg 2019;29:2721-30.

57. Quan Y, Huang A, Ye M, et al. Efficacy of Laparoscopic Mini Gastric Bypass for Obesity and Type 2 Diabetes Mellitus: A Systematic Review and Meta-Analysis. Gastroenterol Res Pract 2015;2015:152852.

58. Dardzińska JA, Kaska Ł, Wiśniewski P, et al. Fasting and post-prandial peptide YY levels in obese patients before and after mini versus Roux-en-Y gastric bypass. Minerva Chir 2017;72:24-30.

59. Kular KS, Manchanda N, Cheema GK. Seven Years of Mini-Gastric Bypass in Type II Diabetes Patients with a Body Mass Index <35 kg/m². Obes Surg 2016;26:1457-62.

60. Solouki A, Kermansaravi M, Davarpanah Jazi AH, et al. One-anastomosis gastric bypass as an alternative procedure of choice in morbidly obese patients. J Res Med Sci 2018;23:84.

61. Robert M, Espalieu P, Pelascini E, et al. Efficacy and safety of one anastomosis gastric bypass versus Roux-en-Y gastric bypass for obesity (YOMEGA): a multicentre, randomised, open-label, non-inferiority trial. Lancet 2019;393:1299-309.

Cite this article as: Aleman R, Lo Menzo E, Szomstein S, Rosenthal RJ. Efficiency and risks of one-anastomosis gastric bypass. Ann Transl Med 2020;8(Suppl 1):S7. doi:10.21037/ atm.2020.02.03 